\title{
Global Fit to Modified Neutrino Couplings and the Cabibbo-Angle Anomaly
}

\author{
Antonio M. Coutinho* \\ Paul Scherrer Institut, $\mathrm{CH}-5232$ Villigen PSI, Switzerland \\ Andreas Crivellin ${ }^{\dagger}$ and Claudio Andrea Manzari $\oplus^{\ddagger}$ \\ Paul Scherrer Institut, CH-5232 Villigen PSI, Switzerland and Physik-Institut, Universität Zürich, \\ Winterthurerstrasse 190, CH-8057 Zürich, Switzerland
}

(Received 13 January 2020; revised 5 May 2020; accepted 6 July 2020; published 14 August 2020)

\begin{abstract}
Recently, discrepancies of up to $4 \sigma$ between the different determinations of the Cabibbo angle were observed. In this context, we point out that this "Cabibbo-angle anomaly" can be explained by lepton flavor universality violating new physics in the neutrino sector. However, modified neutrino couplings to standard model gauge bosons also affect many other observables sensitive to lepton flavor universality violation, which have to be taken into account in order to assess the viability of this explanation. Therefore, we perform a model-independent global analysis in a Bayesian approach and find that the tension in the Cabibbo angle is significantly reduced, while the agreement with other data is also mostly improved. In fact, nonzero modifications of electron and muon neutrino couplings are preferred at more than

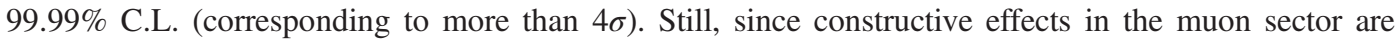
necessary, simple models with right-handed neutrinos (whose global fit we update as a by-product) cannot fully explain data, pointing towards more sophisticated new physics models.
\end{abstract}

DOI: 10.1103/PhysRevLett.125.071802

Introduction.-The standard model (SM) of particle physics has been established with increasing precision within the last decades. In particular, both the electroweak (EW) fit [1-3] and the global fit [4,5] of the CabibboKobayashi-Maskawa (CKM) matrix [6,7] are mainly in good agreement with the SM hypothesis and no new particles were directly observed at the LHC $[8,9]$. Still, there are tensions between the different determinations of the Cabibbo angle from the CKM elements $V_{u s}$ and $V_{u d}$, which became more pronounced recently. Here, $V_{u s}$ from tau decays $[10,11]$ and $V_{u s}$ from kaon decays [12] do not perfectly agree. Furthermore, there is a $\sim 3-4 \sigma$ tension between these determinations and the one from $V_{u d}$ entering superallowed $\beta$ decay (using CKM unitarity) with nonnegligible dependence on the theory predictions [13-16]. In more detail, the different determinations of $V_{u s}$ are as follows: (i) measurements of $K \rightarrow \pi \ell \nu$ together with the form factor $f_{+}(0)$ evaluated at zero momentum transfer result in $V_{u s}=0.2232$ (11) [12]. (ii) $K \rightarrow \ell \nu / \pi \rightarrow \ell \nu$ determines $V_{u s} / V_{u d}$ once the ratio of decay constants $f_{K^{ \pm}} / f_{\pi^{ \pm}}$is known. Using CKM unitarity this results in $V_{u s}=0.22534(44)$ [12]. (iii) $V_{u d}$ is measured via

Published by the American Physical Society under the terms of the Creative Commons Attribution 4.0 International license. Further distribution of this work must maintain attribution to the author(s) and the published article's title, journal citation, and DOI. Funded by SCOAP ${ }^{3}$. super-allowed nuclear $\beta$ decay. Here $V_{u s}$ is again determined via CKM unitarity and using the theory input of Marciano et al. [16] one finds $V_{u s}=0.22699(77)$, while the evaluation of Seng et al. gives $V_{u s}=0.22780(59)$ [14]. (iv) $V_{u s} / V_{u d}$ is also measured in $\tau \rightarrow K \nu, \tau \rightarrow K \nu / \tau \rightarrow \pi \nu$

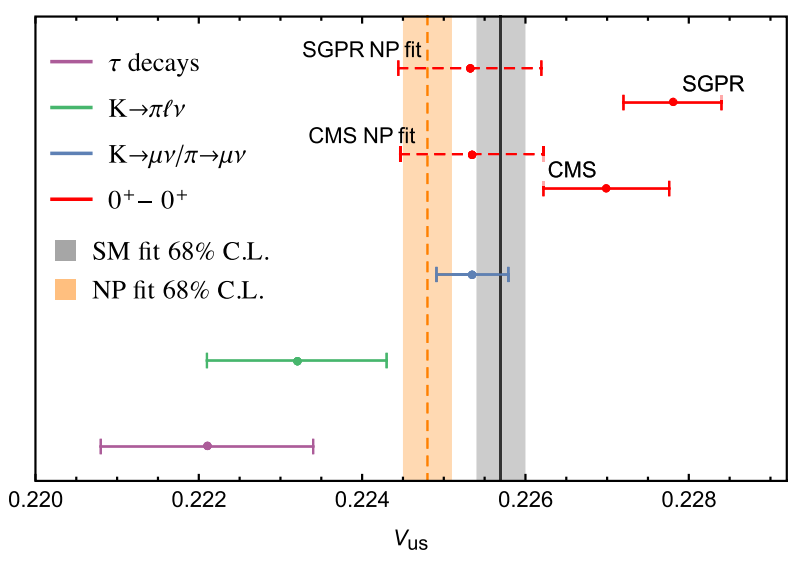

FIG. 1. Measurements of $V_{u s}$ from $\tau$ decays, $K \rightarrow \pi \ell \nu$, $K \rightarrow \mu \nu / \pi \rightarrow \mu \nu$, and $0^{+}-0^{+}$transition using CKM unitarity to convert $V_{u d}$ to $V_{u s}$. The grey band shows the $68 \%$ C.L. posterior within the SM while the orange band corresponds to the NP fit with nonzero values of $\varepsilon_{i i}$. Here SGPR (CMS) stands for the $V_{u s}$ values extracted from superallowed beta decays using the theory input of Ref. [13] (Ref. [16]). Accidentally, the posterior of $V_{u s}$ is the same, independently of the theory input used for beta decays (to the numerical accuracy at which we are working). 
and via inclusive tau decays. Here the HFLAV average is $V_{u s}=0.2221(13)[11]$.

This situation is graphically depicted in Fig. 1. One can clearly see that these measurements are not consistent with each other, and Ref. [17] quantifies this inconsistency to be at the level of $3.6 \sigma(5.1 \sigma)$ if the theory input of Ref. [16] (Ref. [14]) for superallowed beta decay is used.

It is, therefore, very interesting to explore if new physics (NP) can explain this "Cabibbo-angle anomaly." First of all, note that the absolute size of a NP effect potentially capable of explaining this anomaly is quite large since the corresponding SM contribution is generated at tree level and is at most suppressed by one power of the Wolfenstein parameter. Because of this, at the level of effective operators, and given the strong LHC bounds on NP generating two-quarktwo-lepton operators [18], NP entering via four-fermion operators seems to be a disfavored option. Another possibility is a modification of $W$-fermion couplings, where a right-handed $W$ coupling to quarks only improves the fit mildly [17]. Furthermore, a modification of left-handed $W$ couplings to quarks (which is equivalent to an apparent violation of CKM unitarity) can improve the agreement between superallowed beta decay and $V_{u s}$ from kaon decays [19], but generates potentially dangerous effects in other flavor observables (like kaon mixing). Therefore, we will follow a different and novel avenue in this Letter and study the impact of modified (flavor dependent) $W$-boson couplings to neutrinos.

Modified couplings of neutrinos to the SM $W$ are generated via higher dimensional operators in an EFT approach. Here, due to $\mathrm{SU}(2)_{L}$ gauge invariance, in general not only $W$-neutrino couplings but also $Z$-neutrino couplings are modified. Moreover, these modified couplings not only enter $Z$ and $W$ decays, but also all low energy observables involving neutrinos. In particular, ratios testing lepton flavor universality (LFU) in $K, \pi$ and $W$ decays are most relevant due to their exquisite experimental and theoretical precision. There are stringent bounds from $K \rightarrow \mu \nu / K \rightarrow e \nu$ [20,21], $\pi \rightarrow \mu \nu / \pi \rightarrow e \nu$ [22,23], as well as from $\tau \rightarrow \mu \nu \nu / \tau \rightarrow e \nu \nu$ or $W \rightarrow \mu \nu / W \rightarrow e \nu$ [24]. Correlated effects arise, and it is clear that a global fit to all data is necessary in order to assess consistently the impact of modified neutrino couplings.

Modified neutrino couplings to SM gauge bosons have already been considered in the literature in the context of right-handed neutrinos [25-51] and global fits have also been performed $[52,53]$. However, extensions with righthanded neutrinos lead necessarily to destructive interference with the SM, whereas here we will also be interested in the most general case allowing for an arbitrary phase of the NP contribution. The connection to, and correlations with, the Cabibbo-angle anomaly were not considered before and, in addition, we will use the publicly available HEPFIT software [54] to perform a Bayesian analysis, while previous analyses were based on frequentist inference.
After defining our setup and reviewing the relevant observables together with the corresponding NP modification in the next section, we will present the results of our fit the analysis section before we conclude in the final section.

Setup and observables.-As outlined in the introduction, we want to assess the impact of modified neutrino couplings to gauge bosons within an EFT approach. For this purpose, we assume that the NP scale is above the EW scale, as suggested by LHC [8,9] and LEP [55] searches. Therefore, $\mathrm{NP}$ interactions must be $\mathrm{SU}(2)_{L}$ gauge invariant and the number of operators is significantly reduced [56]. In fact, at the dimension 6 level, there is just one operator which modifies only the couplings of gauge bosons to neutrinos [56,57], $\bar{L}_{i} \gamma^{\mu} \tau^{I} L_{j} H^{\dagger} i \stackrel{\leftrightarrow}{D}{ }_{\mu} H$, with $\tau^{I}=\left(1,-\sigma_{1},-\sigma_{2},-\sigma_{3}\right)$, where $\sigma_{i}$ are the Pauli matrices (i.e., it is the difference of the two operators $Q_{\varphi \ell}^{(1)}$ and $Q_{\varphi \ell}^{(3)}$ in the basis of Ref. [57], to which we refer the interested reader for details on the conventions). Note that this operator is Hermitian, meaning the diagonal elements are real. In what follows, we conveniently parametrize the effect of a nonzero Wilson coefficient of this operator in such a way that a neutrino entering a gauge coupling carries a (small) modification of $\frac{1}{2} \varepsilon_{i j}$, resulting in shifts in the $W$ and $Z$ Feynman rules,

$$
\begin{aligned}
& \frac{-i g_{2}}{\sqrt{2}} \bar{\ell}_{i} \gamma^{\mu} P_{L} \nu_{j} W_{\mu} \Rightarrow \frac{-i g_{2}}{\sqrt{2}} \bar{\ell}_{i} \gamma^{\mu} P_{L} \nu_{j} W_{\mu}\left(\delta_{i j}+\frac{1}{2} \varepsilon_{i j}\right), \\
& \frac{-i g_{2}}{2 c_{W}} \bar{\nu}_{i} \gamma^{\mu} P_{L} \nu_{j} Z_{\mu} \Rightarrow \frac{-i g_{2}}{2 c_{W}} \bar{\nu}_{i} \gamma^{\mu} P_{L} \nu_{j} Z_{\mu}\left(\delta_{i j}+\varepsilon_{i j}\right) .
\end{aligned}
$$

Here we assumed massless neutrinos and thus suppressed the PMNS matrix in the $W$ vertex.

Let us then, in the following subsections, consider the observables which will be included in our global fit.

Lepton flavor violating decays: Nondiagonal elements of $\varepsilon_{i j}$ lead to charged lepton flavor violation. Here the bounds from radiative lepton decays $\ell_{i} \rightarrow \ell_{f} \gamma$ are most stringent. Using the results of Ref. [58] we obtain

$$
\operatorname{Br}\left(\ell_{i} \rightarrow \ell_{f} \gamma\right)=\frac{m_{\ell_{i}}^{3}}{4 \pi \Gamma_{\ell_{i}}}\left|\frac{10 e}{384 \pi^{2} M_{W}^{2}} \frac{g_{2}^{2}}{2} m_{\ell_{i}} \varepsilon_{f i}\right|^{2},
$$

where we keep only linear terms in $\varepsilon_{i f}$ and neglect the small mass of the outgoing lepton. The current experimental $90 \%$ C.L. limits on lepton flavor violation processes are $4.2 \times 10^{-13}$ and 4.4(3.3) $\times 10^{-8}$ for $\mu \rightarrow e \gamma$ [59] and $\tau \rightarrow$ $\mu(e) \gamma$ [60], respectively, leading to $\left|\varepsilon_{e \mu}\right| \leq 1.4 \times 10^{-5}$ and $\left|\varepsilon_{\mu \tau}\right| \leq 9.4 \times 10^{-3}$ and $\left|\varepsilon_{e \tau}\right| \leq 1.1 \times 10^{-2}$. These limits on the flavor off-diagonal elements can be used directly, as they are unaffected (at leading order in $\varepsilon_{i j}$ ) by other entries $\varepsilon_{i j}$. Furthermore, since flavor off-diagonal elements of $\varepsilon_{i j}$ in flavor conserving processes do not interfere with the SM contributions, they enter only quadratically. Therefore, 
$\varepsilon_{i j}$ with $i \neq j$ can be safely neglected in the following observables.

EW observables: While the measurements of the mass of the $Z$ boson $\left(m_{Z}\right)$ and the fine structure constant $(\alpha)$ are not affected by the modification of the neutrino couplings in Eq. (1), the Fermi constant $\left(G_{F}\right.$, which is determined with a very high precision from the muon lifetime) is. As such, its value, extracted from $\mu \rightarrow e \nu \nu$, depends on the modification of the $W-\ell-\nu$ coupling. Taking into account that $\operatorname{Br}\left(\mu^{+} \rightarrow \mathrm{e}^{+} \nu_{e} \bar{\nu}_{\mu}\right) \sim 1$ we have

$$
\frac{1}{\tau_{\mu}}=\frac{\left(G_{F}^{\mathcal{L}}\right)^{2} m_{\mu}^{5}}{192 \pi^{3}}(1+\Delta q)\left(1+\frac{1}{2} \varepsilon_{e e}+\frac{1}{2} \varepsilon_{\mu \mu}\right)^{2},
$$

where $G_{F}^{\mathcal{L}}$ is the Fermi constant appearing in the Lagrangian and $\Delta q$ includes phase space, QED and hadronic radiative corrections. Thus we find

$$
G_{F}=G_{F}^{\mathcal{L}}\left(1+\frac{1}{2} \varepsilon_{e e}+\frac{1}{2} \varepsilon_{\mu \mu}\right) .
$$

In addition to $G_{F}$, only the total width of the $Z\left(\Gamma_{Z}\right)$ and the number of light neutrino extracted from invisible $Z$ decays $\left(N_{\nu}\right)$ receive direct modifications in the presence of anomalous neutrino couplings. The number of active neutrinos, as extracted from data [61], is given by

$$
\begin{aligned}
\mathrm{N}_{\nu}^{\text {exp }} & =\left(1+\varepsilon_{e e}\right)^{2}+\left(1+\varepsilon_{\mu \mu}\right)^{2}+\left(1+\varepsilon_{\tau \tau}\right)^{2}, \\
& =2.9840 \pm 0.0082,
\end{aligned}
$$

which in turn also changes $\Gamma_{Z}$, to which it contributes.

We included the modifications of these observables into the EW implementations of HEPFIT [54]; see the Supplemental Material [62], which includes Refs. [63-83], for further details.

Test of LFU: In case the diagonal elements of $\varepsilon_{i i}$ differ from each other, observables testing LFU provide stringent constraints. Here, we have ratios of $W$ decays $\left(W \rightarrow \ell_{i} \nu / W \rightarrow \ell_{j} \nu\right)$ as well as of kaon, pion, and tau decays (see Ref. [84] for an overview). Concerning $B$ decays, only $B \rightarrow D^{(*)} e \nu / B \rightarrow D^{(*)} \mu \nu$ provides a relevant constraint [85]. The corresponding observables, including their dependence on $\varepsilon_{i i}$, are shown in Table I of the Supplemental Material [62]. For tau decays, we include their correlations as given in Ref. [11].

Determination of $\left|V_{u s}\right|$ : We can now turn to the determination of $V_{u s}$ as already briefly depicted in the introduction (see Fig. 1).

$K_{\ell 3}: V_{u s}$ can be determined from the semileptonic kaon decays. In order to allow for LFU violation, one has to separate muon from electron modes. Averaging $K_{L}, K^{ \pm}$, and $K_{S}$ modes [23], one finds

$$
\left|V_{u s}^{K_{\mu 3}}\right| \simeq 0.2234(8), \quad\left|V_{u s}^{K_{e 3}}\right| \simeq 0.2230(21),
$$

by using the lattice average [12] of the form factor at zero momentum transfer $f_{+}(0)=0.9698(17),\left(N_{f}=2+1+1\right)$. We choose to include the muon mode in the global fit, while the electron mode is already taken into account via the LFU ratios in Table I of the Supplemental Material [62]. The NP modification, including the indirect effect of $G_{F}$, is

$$
\left|V_{u s}^{K_{\mu 3}}\right| \simeq\left|V_{u s}^{\mathcal{L}}\right|\left(1-\frac{1}{2} \varepsilon_{e e}\right) .
$$

$K_{\ell 2}: \operatorname{Br}\left(K^{ \pm} \rightarrow \mu^{ \pm} \nu\right) / \operatorname{Br}\left(\pi^{ \pm} \rightarrow \mu^{ \pm} \nu\right)$ determines $V_{u s} / V_{u d}$. Including long-distance electromagnetic and strong isospin breaking corrections [86] and using the average of the lattice determinations for the ratio of form factors [12] $f_{K^{ \pm}} / f_{\pi^{ \pm}}=1.1967(18)$, with $\left(N_{f}=2+1+1\right)$, we find

$$
\left|V_{u s}^{K / \pi}\right| \simeq 0.22535(44)
$$

where we assumed CKM unitarity and took $\left|V_{u b}\right| \simeq 0.004$. Note that the value of $V_{u s}$ is very insensitive to $V_{u b}$, whose uncertainty can therefore be neglected, and that this $V_{u b}$ determination is not affected by $\varepsilon_{i j}$.

$0^{+}-0^{+}$transitions: $\left|V_{u d}\right|$ can be extracted from superallowed nuclear $\beta$ transitions [87]. The result relies heavily on the evaluation of radiative corrections. We consider the two different results (as suggested in Ref. [17]) of Marciano et al. [16] (CMS) and Seng et al. [14] (SGPR), which produce

$$
\left|V_{u s}\right|_{\mathrm{CMS}}=0.22699(77), \quad\left|V_{u s}\right|_{\mathrm{SGPR}}=0.22780(60),
$$

where CKM unitarity was again used. Turning on the NP couplings, we find the following modification

$$
\left|V_{u s}^{\beta}\right| \simeq \sqrt{1-\left|V_{u d}^{\mathcal{L}}\right|^{2}\left(1-\frac{1}{2} \varepsilon_{\mu \mu}\right)^{2}} .
$$

$\tau$ decays: $\left|V_{u s}\right|$ can be also determined from hadronic $\tau$ decays [11]. Here the average is [11] $\left|V_{u s}^{\tau}\right|=0.2221(13)$. Both $\tau \rightarrow K \nu / \tau \rightarrow \pi \nu$ and the inclusive mode measure $V_{u s} / V_{u d}$, which means there is, at leading order, no dependence on $\varepsilon_{i j}$, and the determination is then unaffected by our NP contributions. This is different for the determination from $\tau \rightarrow K \nu$, whose dependence on $\varepsilon_{i j}$ is given by

$$
\left|V_{u s}^{\tau \rightarrow K \nu}\right| \simeq\left|V_{u s}^{\mathcal{L}}\right|\left(1-\frac{1}{2} \varepsilon_{e e}-\frac{1}{2} \varepsilon_{\mu \mu}+\frac{1}{2} \varepsilon_{\tau \tau}\right) .
$$

Since this mode as well as other hadronic tau decays are already included in the LFU ratios, we do not include the $V_{u s}$ from tau decays in our global fit. Nevertheless, we can still predict the change in $V_{u s}^{\tau \rightarrow K \nu}$.

Analysis. - In this section we perform the global fit to the modified neutrino couplings [see Eq. (1)], taking into account the observables discussed in the previous section. Before presenting the results, let us briefly discuss the statistical inference procedure we adopted. Our analysis is 
performed in a Bayesian framework using the publicly available HEPFIT package [54], whose Markov chain Monte Carlo (MCMC) determination of posteriors is powered by the Bayesian Analysis Toolkit (BAT) [88].

In order not to overweight the $V_{u d}$ measurements from $0^{+}-0^{+}$transitions, we do not include both theory determinations at the same time, but rather define two scenarios: NP-I with $\left|V_{u d}\right|_{\text {CMS }}$ from Ref. [16], and NP-II with $\left|V_{u d}\right|_{\text {SGPR }}$ from Ref. [13]. Bayesian model comparison between different scenarios can be accomplished by evaluating an information criterion (IC) $[89,90]$. This quantity is characterized by the mean and the variance of the posterior of the $\log$-likelihood, $\log \mathcal{L}$, which yield an estimate of the predictive accuracy of the model [91], and a penalty factor for the number of free parameters fitted. Preference for a model is given according to the smallest IC value, following the scale of evidence suggested in Refs. [92,93]. The full list of fit parameters and details on the choice of priors can be found in Supplemental Material [62].

Let us now probe the impact of nonzero values of $\varepsilon_{i j}$. As noted in the last section, one can neglect the flavor offdiagonal elements whose values are directly bounded by radiative lepton decays. As such, in the global fit we only have to consider $\varepsilon_{e e}, \varepsilon_{\mu \mu}$, and $\varepsilon_{\tau \tau}$. The $68 \%$ C.L. intervals for fit parameters of the flavor sector $\left(V_{u s}^{\mathcal{L}}, \varepsilon_{i i}\right)$ within the two NP scenarios can be found in the Supplemental Material [62]. One can see that there is only a mild difference between both scenarios. In particular, the posterior of $V_{u s}^{\mathcal{L}}$ is accidentally even the same and only the preferred region for $\varepsilon_{e e}\left(\varepsilon_{\mu \mu}\right)$ in scenario NP-II is slightly more negative (positive) than in scenario NP-I. Therefore, we only present the results for the two-dimensional $\varepsilon_{i i}-\varepsilon_{j j}$ planes in Fig. 2 within scenario NP-II (scenario NP-I is approximately $1 \sigma$ more compatible with the SM hypothesis). There, the $68 \%, 95 \%$, and 99\% C.L. contours are shown, and it is clear from the $\varepsilon_{e e^{-}} \varepsilon_{\mu \mu}$ plane, where the largest deviation from SM can be found, that these regions do not overlap with the SM point $\varepsilon_{i i}=0$, and that $\varepsilon_{e e}$ and $\varepsilon_{\mu \mu}$ possess an anticorrelation.

Concerning the $V_{u s}$ determination, we have also depicted the posterior of scenario NP-II and the updated values extracted from superallowed beta decay in Fig. 1. In summary, the main drivers leading to a better fit of the NP scenarios are the $V_{u s}$ determination from super-allowed beta decays, $\tau \rightarrow \mu \nu \nu / \mu \rightarrow e \nu \nu$ and $\tau \rightarrow \mu \nu \nu / \tau \rightarrow e \nu \nu$ [62].

For a more direct model comparison between the NP fits and the SM we look at the IC values. Here we obtain for the $\mathrm{SM} \mathrm{IC}_{\mathrm{SM}}=73$, compared to $\mathrm{IC}_{\mathrm{NP}-\mathrm{II}}=63$ and $\mathrm{IC}_{\mathrm{NP}-\mathrm{I}}=60$ for the two NP scenarios. In the vein of Ref. [93], this constitutes "very strong" evidence against the SM, further evidencing that current data clearly favors the NP hypothesis, and promoting the search for a NP model.

For that UV complete NP explanation obviously the possibility of right-handed neutrinos comes to mind, as

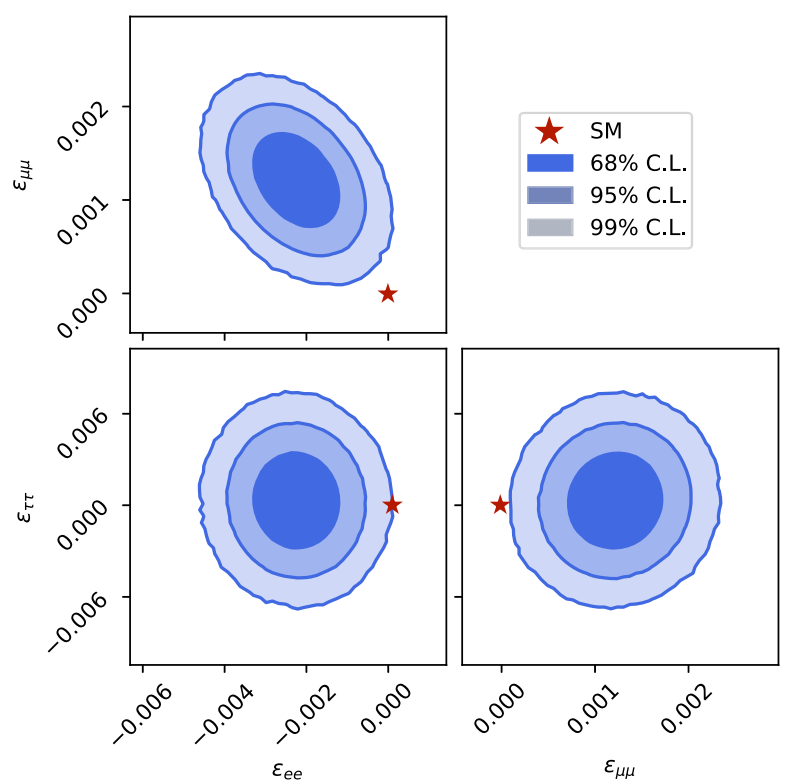

FIG. 2. Global fit for scenario NP-II. The 2D fit $(68 \%, 95 \%$, and $99 \%$ C.L.) for $\varepsilon_{i i}-\varepsilon_{j j}$ as well as the 1D fit for each $\varepsilon_{i i}$ (68\% C.L. indication) are shown.

these models give tree-level effects in $Z-\nu-\nu$ and $W-\ell-\nu$ couplings. However, here the effect is necessarily destructive (i.e., $\varepsilon_{i i}<0$ ), which is not in agreement with the preferred regions found in our fit. Nonetheless, performing the fit we find: $\varepsilon_{e e}=-0.0013_{-0.0006}^{+0.0006}, \varepsilon_{\tau \tau}=-0.0014_{-0.0015}^{+0.0010}$, and $0>\varepsilon_{\mu \mu}>-0.0002$ at $68 \%$ C.L. The shift towards values compatible with zero (within $\sim 2.2 \sigma$ for $\varepsilon_{e e}$, and $1.2 \sigma$ for $\varepsilon_{\tau \tau}$ ) signals a feeble improvement with respect to the SM. In fact, such a conclusion is supported by an IC value of 78, which is even bigger than the one of the SM due to the penalty for extra parameters. Moreover, once the constraint from $\mu \rightarrow e \gamma$, arising in models with $\varepsilon_{e \mu}=$ $\sqrt{\varepsilon_{e e} \varepsilon_{\mu \mu}}[46,94-101]$ is taken into account, it is even more difficult in this scenario to explain data well.

Since right-handed neutrinos cannot fully explain the tensions within the EW fit, naturally the quest for a different UV completion arises. Even though a complete analysis is beyond the scope of this work, note that this can be achieved, e.g., by adding additional vectorlike leptons (VLLs) which induce tree-level modifications to $Z$ and $W$ couplings with leptons after EW symmetry breaking. Here, $\mathrm{SU}(2)$ singlets and triplets generate the desired Wilson coefficients $C_{\phi \ell}^{(1,3)}$, while $\mathrm{SU}(2)$ doublets modify couplings of right-handed charged leptons to $Z$. One has, therefore, four VLLs at our disposal: two doublets and two singlets which differ in hypercharge and contribute as

$$
\begin{array}{ll}
N: C_{\phi \ell}^{(3) f i}=-C_{\phi \ell}^{(1) f i}<0, & E: C_{\phi \ell}^{(3) f i}=C_{\phi \ell}^{(1) f i}<0, \\
\Sigma_{0}: C_{\phi \ell}^{(3) f i}=\frac{1}{3} C_{\phi \ell}^{(1) f i}>0, \quad \Sigma_{1}: C_{\phi \ell}^{(3) f i}=-\frac{1}{3} C_{\phi \ell}^{(1) f i}>0,
\end{array}
$$


using the conventions of Ref. [102]. It is thus clear that we can produce any combination of $C_{\phi \ell}^{(1,3)}$ with arbitrary sign, including $C_{\phi \ell}^{(1)}=-C_{\phi \ell}^{(3)}$ being positive or negative. Such a linear combination of vectorlike leptons might seem ad hoc at first glance. However, this is exactly what happens in composite or extradimensional models with custodial protection [103,104] [see, e.g., Refs. [105,106] for a generalization to the lepton sector with $\mathrm{SU}(2)_{L}$ triplets]. In such models, the symmetry group is chosen in such a way that the VLL representations (generated for instance as Kaluza-Klein excitations) lead to modifications of $Z-\nu-\nu$ and $W-\ell-\nu$ couplings, but not to $Z-\ell-\ell$ interactions. As this corresponds exactly to the case at hand, $C_{\phi \ell}^{(1)}=-C_{\phi \ell}^{(3)}$, extradimensional or composite models with custodial protection can therefore very well give rise to the scenario obtained in our model-independent fit.

Conclusions and Outlook.-In this Letter, we performed a model independent global fit to modified neutrino couplings motivated by the Cabbibo-angle anomaly (i.e., the disagreement between the different determinations of $V_{u s}$ ). Taking into account all relevant observables related to the EW sector of the SM and observables testing LFU (like $\tau \rightarrow \mu \nu \nu / \tau \rightarrow e \nu \nu, \pi \rightarrow \mu \nu \nu / \pi \rightarrow e \nu \nu$, etc.), we found that agreement with data can be significantly improved by small modifications $\varepsilon_{i i}$. Our results for this NP scenario are depicted in Fig. 2, showing the SM hypothesis lies beyond the $99.99 \%$ C.L. region, corresponding to a deviation of more than $4 \sigma$. Furthermore, the IC values of the scenarios here considered strongly prefer the NP hypothesis.

However, conventional models with right-handed neutrinos, which lead to necessary destructive interference, cannot explain data very well. Nevertheless, since these models are well motivated by the observed nonvanishing neutrino masses, we updated their global fit, taking into account the different $V_{u s}$ determinations.

Clearly, more data and further theory input is needed to clarify the situation in the future. Also, the study and construction of NP models which can give a constructive effect in $Z-\nu-\nu$ and $W-\ell-\nu$ couplings, in particular strongly coupled theories with custodial protection, is a promising direction of research, building upon the results of this article. Furthermore, as our explanation involves flavor-dependent couplings, the Cabibbo-angle anomaly fits into the bigger picture of deviations from LFU as observed in $b \rightarrow s \ell^{+} \ell^{-}$transitions [107-117] and the anomalous magnetic moment of the muon and electron $[58,118]$. This opens up the possibility of so far undiscovered correlations among these observables with UV complete models.

We thank Marco Fedele, Julian Heeck, Martin Hoferichter, Ayan Paul, Hugo M. Proença, and Mauro Valli for useful discussions and/or help with HEPFIT.
The work of A.C. is supported by a Professorship Grant (No. PP00P2_176884) of the Swiss National Science Foundation. A. M. C. acknowledges support by the Swiss National Science Foundation under Contract No. 200021_178967.

*antonio.coutinho@psi.ch

†andreas.crivellin@cern.ch

claudioandrea.manzari@physik.uzh.ch

[1] J. de Blas, M. Ciuchini, E. Franco, S. Mishima, M. Pierini, L. Reina, and L. Silvestrini, J. High Energy Phys. 12 (2016) 135.

[2] J. Haller, A. Hoecker, R. Kogler, K. Mönig, T. Peiffer, and J. Stelzer, Eur. Phys. J. C 78, 675 (2018).

[3] T. A. Aaltonen et al. (CDF and D0 Collaborations), Phys. Rev. D 97, 112007 (2018).

[4] M. Ciuchini, G. D’Agostini, E. Franco, V. Lubicz, G. Martinelli, F. Parodi, P. Roudeau, and A. Stocchi, J. High Energy Phys. 07 (2001) 013.

[5] A. Hocker, H. Lacker, S. Laplace, and F. Le Diberder, Eur. Phys. J. C 21, 225 (2001).

[6] N. Cabibbo, Phys. Rev. Lett. 10, 531 (1963).

[7] M. Kobayashi and T. Maskawa, Prog. Theor. Phys. 49, 652 (1973).

[8] J. N. Butler (CMS Collaboration), Highlights and perspectives from the CMS experiment, in Proceedings of the Fifth Annual Large Hadron Collider Physics conference (LHCP2017) Shanghai, China (2017).

[9] L. Masetti (ATLAS Collaboration), Nucl. Part. Phys. Proc. 303-305, 43 (2018).

[10] A. Lusiani, SciPost Phys. Proc. 1, 001 (2019).

[11] Y.S. Amhis et al. (HFLAV Collaboration), arXiv: 1909.12524.

[12] S. Aoki et al. (Flavour Lattice Averaging Group), Eur. Phys. J. C 80, 113 (2020).

[13] C.-Y. Seng, M. Gorchtein, H. H. Patel, and M. J. Ramsey-Musolf, Phys. Rev. Lett. 121, 241804 (2018).

[14] C. Y. Seng, M. Gorchtein, and M. J. Ramsey-Musolf, Phys. Rev. D 100, 013001 (2019).

[15] M. Gorchtein, Phys. Rev. Lett. 123, 042503 (2019).

[16] A. Czarnecki, W. J. Marciano, and A. Sirlin, Phys. Rev. D 100, 073008 (2019).

[17] Y. Grossman, E. Passemar, and S. Schacht, J. High Energy Phys. 07 (2020) 068.

[18] M. Aaboud et al. (ATLAS Collaboration), J. High Energy Phys. 10 (2017) 182.

[19] B. Belfatto, R. Beradze, and Z. Berezhiani, Eur. Phys. J. C 80, 149 (2020).

[20] F. Ambrosino et al. (KLOE Collaboration), Eur. Phys. J. C 64, 627 (2009); 65, 703(E) (2010).

[21] C. Lazzeroni et al. (NA62 Collaboration), Phys. Lett. B 719, 326 (2013).

[22] A. Aguilar-Arevalo, M. Aoki, M. Blecher, D. I. Britton, D. A. Bryman et al. (PiENu Collaboration), Phys. Rev. Lett. 115, 071801 (2015).

[23] M. Tanabashi et al. (Particle Data Group), Phys. Rev. D 98, 030001 (2018). 
[24] J. Alcaraz et al. (ALEPH, DELPHI, L3, OPAL Collaborations and LEP Electroweak Working Group), arXiv:hep-ex/0612034.

[25] B. W. Lee and R. E. Shrock, Phys. Rev. D 16, 1444 (1977).

[26] R. E. Shrock, Phys. Lett. 96B, 159 (1980).

[27] J. Schechter and J. W. F. Valle, Phys. Rev. D 22, 2227 (1980).

[28] R. E. Shrock, Phys. Rev. D 24, 1232 (1981).

[29] R. E. Shrock, Phys. Rev. D 24, 1275 (1981).

[30] P. Langacker and D. London, Phys. Rev. D 38, 886 (1988).

[31] S. M. Bilenky and C. Giunti, Phys. Lett. B 300, 137 (1993).

[32] E. Nardi, E. Roulet, and D. Tommasini, Phys. Lett. B 327, 319 (1994).

[33] D. Tommasini, G. Barenboim, J. Bernabeu, and C. Jarlskog, Nucl. Phys. B444, 451 (1995).

[34] S. Bergmann and A. Kagan, Nucl. Phys. B538, 368 (1999).

[35] W. Loinaz, N. Okamura, T. Takeuchi, and L.C.R. Wijewardhana, Phys. Rev. D 67, 073012 (2003).

[36] W. Loinaz, N. Okamura, S. Rayyan, T. Takeuchi, and L. C. R. Wijewardhana, Phys. Rev. D 68, 073001 (2003).

[37] W. Loinaz, N. Okamura, S. Rayyan, T. Takeuchi, and L. C. R. Wijewardhana, Phys. Rev. D 70, 113004 (2004).

[38] S. Antusch, C. Biggio, E. Fernandez-Martinez, M. B. Gavela, and J. Lopez-Pavon, J. High Energy Phys. 10 (2006) 084.

[39] S. Antusch, J. P. Baumann, and E. Fernandez-Martinez, Nucl. Phys. B810, 369 (2009).

[40] C. Biggio, Phys. Lett. B 668, 378 (2008).

[41] R. Alonso, M. Dhen, M. B. Gavela, and T. Hambye, J. High Energy Phys. 01 (2013) 118.

[42] A. Abada, D. Das, A. M. Teixeira, A. Vicente, and C. Weiland, J. High Energy Phys. 02 (2013) 048.

[43] E. Akhmedov, A. Kartavtsev, M. Lindner, L. Michaels, and J. Smirnov, J. High Energy Phys. 05 (2013) 081.

[44] L. Basso, O. Fischer, and J. J. van der Bij, Europhys. Lett. 105, 11001 (2014).

[45] A. Abada, A. M. Teixeira, A. Vicente, and C. Weiland, J. High Energy Phys. 02 (2014) 091.

[46] S. Antusch and O. Fischer, J. High Energy Phys. 10 (2014) 094.

[47] S. Antusch and O. Fischer, J. High Energy Phys. 05 (2015) 053.

[48] A. Abada, V. De Romeri, and A. M. Teixeira, J. High Energy Phys. 02 (2016) 083.

[49] A. Abada and T. Toma, J. High Energy Phys. 02 (2016) 174.

[50] A. Abada and T. Toma, J. High Energy Phys. 08 (2016) 079.

[51] P. D. Bolton, F. F. Deppisch, and P. S. B. Dev, J. High Energy Phys. 03 (2020) 170.

[52] E. Fernandez-Martinez, J. Hernandez-Garcia, and J. Lopez-Pavon, J. High Energy Phys. 08 (2016) 033.

[53] M. Chrzaszcz, M. Drewes, T. E. Gonzalo, J. Harz, S. Krishnamurthy, and C. Weniger, Eur. Phys. J. C 80, 569 (2020).

[54] J. De Blas et al., Eur. Phys. J. C 80, 456 (2020).

[55] M. Antonelli and S. Moretti, Ital. Phys. Soc. Proc. 78, 45 (2002), https://inspirehep.net/literature/559289.
[56] W. Buchmuller and D. Wyler, Nucl. Phys. B268, 621 (1986).

[57] B. Grzadkowski, M. Iskrzynski, M. Misiak, and J. Rosiek, J. High Energy Phys. 10 (2010) 085.

[58] A. Crivellin, M. Hoferichter, and P. Schmidt-Wellenburg, Phys. Rev. D 98, 113002 (2018).

[59] A. M. Baldini et al. (MEG Collaboration), Eur. Phys. J. C 76, 434 (2016).

[60] B. Aubert et al. (BABAR Collaboration), Phys. Rev. Lett. 104, 021802 (2010).

[61] S. Schael et al. (ALEPH, DELPHI, L3, OPAL, SLD Collaborations, LEP Electroweak Working Group, SLD Electroweak Group, and SLD Heavy Flavour Group), Phys. Rep. 427, 257 (2006).

[62] See the Supplemental Material at http://link.aps.org/ supplemental/10.1103/PhysRevLett.125.071802 for details on the fit.

[63] S. Schael et al. (ALEPH, DELPHI, L3, OPAL Collaborations and LEP Electroweak Working Group), Phys. Rep. 532, 119 (2013).

[64] D. M. Webber, V. Tishchenko, Q. Peng, S. Battu, R. M. Carey et al. (MuLan Collaboration), Phys. Rev. Lett. 106, 041803 (2011); 106, 079901(E) (2011).

[65] M. Aaboud et al. (ATLAS Collaboration), Phys. Lett. B 784, 345 (2018).

[66] CMS Collaboration, CMS-PAS-HIG-19-004, 2019, https://inspirehep.net/literature/1780985.

[67] T. E. W. Group and T. Aaltonen (CD and D0) (2016).

[68] M. Aaboud et al. (ATLAS Collaboration), Eur. Phys. J. C 79, 290 (2019).

[69] A. M. Sirunyan et al. (CMS Collaboration), Eur. Phys. J. C 79, 313 (2019).

[70] V. Cirigliano and I. Rosell, Phys. Rev. Lett. 99, 231801 (2007), arXiv:0707.3439.

[71] G. Czapek, A. Federspiel, A. Fluckiger, D. Frei, B. Hahn et al., Phys. Rev. Lett. 70, 17 (1993).

[72] D. I. Britton et al., Phys. Rev. Lett. 68, 3000 (1992).

[73] D. A. Bryman, R. Dubois, T. Numao, B. Olaniyi, A. Olin, M. S. Dixit, D. Berghofer, J. M. Poutissou, J. A. Macdonald, and B. C. Robertson, Phys. Rev. Lett. 50, 7 (1983).

[74] M. Antonelli et al. (FlaviaNet Working Group on Kaon Decays), Eur. Phys. J. C 69, 399 (2010).

[75] V. Cirigliano, G. Ecker, H. Neufeld, A. Pich, and J. Portoles, Rev. Mod. Phys. 84, 399 (2012), arXiv: 1107.6001

[76] M. Awramik, M. Czakon, A. Freitas, and G. Weiglein, Phys. Rev. D 69, 053006 (2004).

[77] A. Sirlin, Phys. Rev. D 22, 971 (1980).

[78] M. Faisst, J. H. Kuhn, T. Seidensticker, and O. Veretin, Nucl. Phys. B665, 649 (2003).

[79] L. Avdeev, J. Fleischer, S. Mikhailov, and O. Tarasov, Phys. Lett. B 336, 560 (1994); 349, 597(E) (1995).

[80] K. G. Chetyrkin, J.H. Kuhn, and M. Steinhauser, Phys. Lett. B 351, 331 (1995).

[81] K. G. Chetyrkin, J.H. Kuhn, and M. Steinhauser, Phys. Rev. Lett. 75, 3394 (1995).

[82] A. Broncano, M. B. Gavela, and E. E. Jenkins, Phys. Lett. B 552, 177 (2003); 636, 332(E) (2006).

[83] A. Abada, C. Biggio, F. Bonnet, M. B. Gavela, and T. Hambye, J. High Energy Phys. 12 (2007) 061. 
[84] A. Pich, Prog. Part. Nucl. Phys. 75, 41 (2014).

[85] M. Jung and D. M. Straub, J. High Energy Phys. 01 (2019) 009.

[86] V. Cirigliano and H. Neufeld, Phys. Lett. B 700, 7 (2011).

[87] J. Hardy and I. S. Towner, Proc. Sci., CKM2016 (2016) 028.

[88] A. Caldwell, D. Kollar, and K. Kroninger, Comput. Phys. Commun. 180, 2197 (2009).

[89] T. Ando, Biometrika 94, 443 (2007).

[90] T. Ando, Am. J. Math. Manag. Sci. 31, 13 (2011).

[91] A. Gelman, J. Hwang, and A. Vehtari, arXiv:1307.5928.

[92] H. Jeffreys, The Theory of Probability, 3rd ed., Oxford Classic Texts in the Physical Sciences (Oxford University Press, England, 1998).

[93] R. E. Kass and A. E. Raftery, J. Am. Stat. Assoc. 90, 773 (1995).

[94] R. N. Mohapatra and J. W. F. Valle, Phys. Rev. D 34, 1642 (1986).

[95] J. Bernabeu, A. Santamaria, J. Vidal, A. Mendez, and J. W. F. Valle, Phys. Lett. B 187, 303 (1987).

[96] G. C. Branco, W. Grimus, and L. Lavoura, Nucl. Phys. B312, 492 (1989).

[97] W. Buchmuller and D. Wyler, Phys. Lett. B 249, 458 (1990).

[98] A. Pilaftsis, Z. Phys. C 55, 275 (1992).

[99] P. S. Bhupal Dev and A. Pilaftsis, Phys. Rev. D 86, 113001 (2012).

[100] M. Malinsky, J. C. Romao, and J. W. F. Valle, Phys. Rev. Lett. 95, 161801 (2005).

[101] R. Coy and M. Frigerio, Phys. Rev. D 99, 095040 (2019).

[102] J. de Blas, J. Criado, M. Perez-Victoria, and J. Santiago, J. High Energy Phys. 03 (2018) 109.

[103] K. Agashe, A. Delgado, M. J. May, and R. Sundrum, J. High Energy Phys. 08 (2003) 050.
[104] K. Agashe, R. Contino, L. Da Rold, and A. Pomarol, Phys. Lett. B 641, 62 (2006).

[105] F. del Aguila, A. Carmona, and J. Santiago, J. High Energy Phys. 08 (2010) 127.

[106] A. Carmona and F. Goertz, J. High Energy Phys. 04 (2013) 163.

[107] B. Capdevila, A. Crivellin, S. Descotes-Genon, J. Matias, and J. Virto, J. High Energy Phys. 01 (2018) 093.

[108] W. Altmannshofer, P. Stangl, and D. M. Straub, Phys. Rev. D 96, 055008 (2017).

[109] G. D'Amico, M. Nardecchia, P. Panci, F. Sannino, A. Strumia, R. Torre, and A. Urbano, J. High Energy Phys. 09 (2017) 010.

[110] M. Ciuchini, A. M. Coutinho, M. Fedele, E. Franco, A. Paul, L. Silvestrini, and M. Valli, Eur. Phys. J. C 77, 688 (2017).

[111] G. Hiller and I. Nisandzic, Phys. Rev. D 96, 035003 (2017).

[112] L.-S. Geng, B. Grinstein, S. Jäger, J. Martin Camalich, X.-L. Ren, and R.-X. Shi, Phys. Rev. D 96, 093006 (2017).

[113] T. Hurth, F. Mahmoudi, D. Martinez Santos, and S. Neshatpour, Phys. Rev. D 96, 095034 (2017).

[114] M. Algueró, B. Capdevila, A. Crivellin, S. DescotesGenon, P. Masjuan, J. Matias, and J. Virto, Eur. Phys. J. C 79, 714 (2019).

[115] J. Aebischer, W. Altmannshofer, D. Guadagnoli, M. Reboud, P. Stangl, and D. M. Straub, Eur. Phys. J. C 80, 252 (2020).

[116] M. Ciuchini, A. M. Coutinho, M. Fedele, E. Franco, A. Paul, L. Silvestrini, and M. Valli, Eur. Phys. J. C 79, 719 (2019).

[117] A. Arbey, T. Hurth, F. Mahmoudi, D. Martinez Santos, and S. Neshatpour, Phys. Rev. D 100, 015045 (2019).

[118] H. Davoudiasl and W. J. Marciano, Phys. Rev. D 98, 075011 (2018). 